\title{
A REVIEW ON THE POTENTIAL PROPERTIES OF GINSENG
}

\section{Riya Khurana}

Conceptive Farmacia Pvt. Ltd.

Article Info: Received 28 November 2019; Accepted 14 December 2019

DOI: https://doi.org/10.32553/ijmbs.v3i12.803

Corresponding author: Riya Khurana

Conflict of interest: No conflict of interest.

\section{Abstract}

Ginseng is a traditional herb mostly used in eastern world, namely the countries of Korea and China. It is believed to be a herbal elixir and useful in promotion of long healthy life. It is known to increase strength and vital capacity of the body (1). Ginsenosides are the main active compounds that constitute ginseng. They are considered to be steroidal saponins that act as anti- tumor activists (2). They have been known to show a myriad of pharmacological effects on target tissues, although the type of ginsenoside varies in each species of ginseng. This review study aims to explore the history, mechanisms, targets and effect of ginseng.

\section{Introduction}

\subsection{Brief history}

Ginseng is a 2000 year old traditionally used herb to cure an array of disorders. Particularly the part which has the root is known for its potential to enhance physical potential and capacity (3). It is used more as a restorative element for the body than a curative measure (1). Known as the king of herbs, it is described to cure 23 diseases (Encyclopedia of Herbs by Li Shizhen in China).

There are thirteen species of ginseng explored so far.

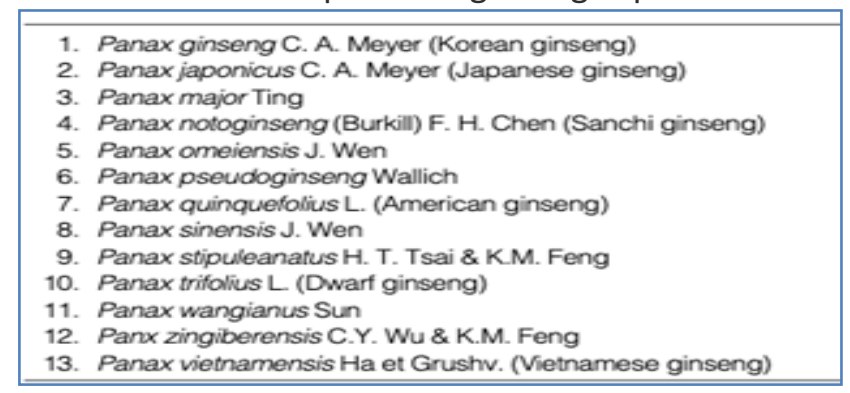

Figure 1: The 13 species of Ginseng, Genus 'Panax' (1)

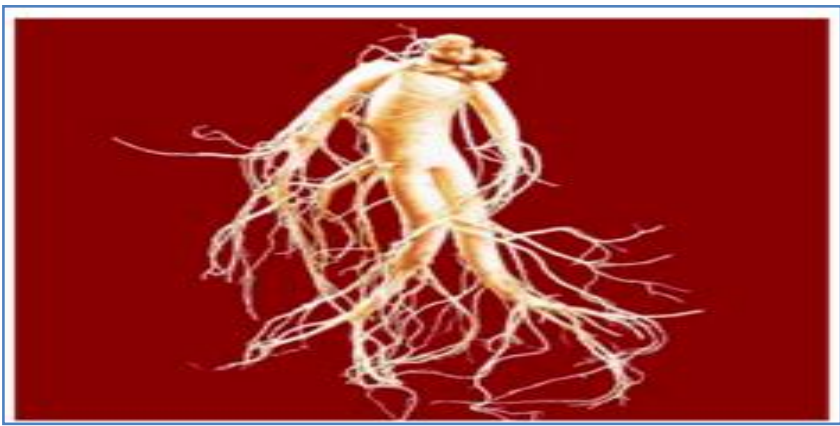

Figure 2: Root of Ginseng (1)

\subsection{Types and structure of Ginseng}

Western world is little skeptic of the medicinal properties and claims of the ginseng. The available form of ginseng in America and Europe is the powder preparation of ginseng root of Panax ginseng or Panax quinquefolius (4). Siberian ginseng is also a type of ginseng coming from Ariliaceae family containing different ginsenoside. "White ginseng" arise from air-drying the roots of the 5-7 years old plant while "Red ginseng" rise from steam- treating the same part for two to four hours (4). Ashwagandha, Withania somnifera is known as Indian ginseng. W.somnifera consists of alkaloids, lactones and withanolides which have the structure similar to the constituents of Panax ginseng i.e. ginsenosides (5).

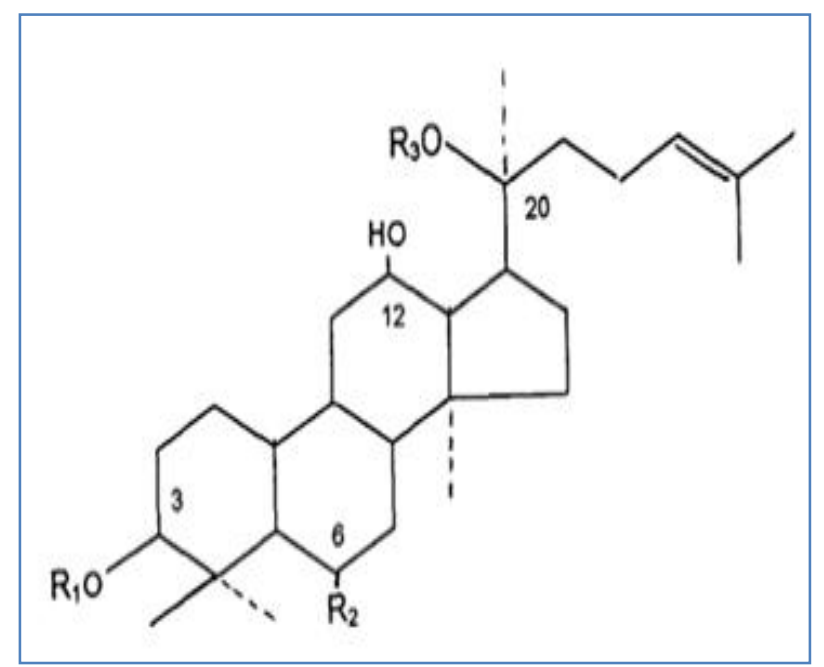




\begin{tabular}{|c|c|c|c|c|}
\hline \multirow[b]{2}{*}{ senoside } & \multicolumn{3}{|c|}{$20(S)$-protopanaxadiols } & \multirow[b]{2}{*}{$\%$ in Roo } \\
\hline & $R_{1}$ & $\mathbf{R}_{2}$ & $\mathbf{R}_{3}$ & \\
\hline & $\mathrm{Glc}^{2}-\mathrm{Glc}$. & $\mathrm{H}$ & $\mathrm{Glc}^{6} \cdot \mathrm{Glc}$ & 0.38 \\
\hline & Glc ${ }^{2}-\mathrm{Glc}$ - & $\mathrm{H}$ & $G c^{6}-A r a(p)$ & 0.13 \\
\hline & $\mathrm{Glc}^{2} \cdot$ Glc- & $\mathrm{H}$ & Glc ${ }^{6}-A r a(f)$ & 0.19 \\
\hline & $\mathrm{Glc}^{2} \cdot \mathrm{Glc}$. & $\mathrm{H}$ & Gle & 0.04 \\
\hline \multirow{6}{*}{ senoside } & 206 & -protopanaxa & atriols & \\
\hline & $\mathbf{R}_{2}$ & $\mathbf{R}_{2}$ & $\mathbf{R}_{3}$ & $\%$ in Root \\
\hline & $\mathrm{H}$ & -O.Glc ${ }^{2}-\mathrm{Rha}$ & Glc- & 0.15 \\
\hline & $\mathrm{H}$ & -O-Glc ${ }^{2}-\mathrm{Glc}$ & $\mathrm{H}: 2 \mathrm{O}(\mathrm{S})$ & 0.09 \\
\hline & $\mathrm{H}$ & O.Glc- & Glc. & 0.38 \\
\hline & $\mathrm{H}$ & -O-Glc ${ }^{2}$ & $\mathrm{H}: 20(\mathrm{~S})$ & 0.02 \\
\hline
\end{tabular}

Figure 3: Ginsenoside structure comprising of its main active compounds (3)

Ginsenosides differ in structure from one another in a way that they have variable attachment sites, sugar fractions and their numbers. Sugar units found in ginsenosides are glucose, saccharose, maltose and fructose which are bound to carbon position 3,6 or 20. The biological activity of the sugar largely depends on its binding site (3). Properties of ginsenosides also vary according to the position of placement of the hydroxyl $(-\mathrm{OH})$ group. Besides this, the number of hydroxyl groups present was also found to affect the pharmacological functions (3). Ginsenosides are structurally different compounds i.e. they exhibit stereochemistry at C-20. isolated ginsenosides have been found to be present in the form of enantiomers (6). After the studies and researchers more than 20 ginsenosides have been found which are obtained from flowers, roots or leaves of the ginseng plant. The quantification of chemical components have been done with the help of HPLC and mass spectrophotometry (4). Not every part of the ginseng root produces the same amount and quality of extract. The quantity of ginsenosides vary, depending on the main and fine roots. The ginseng extract is mainly prepared from the epidermal part of the plant root which consist of lateral and fine roots due to the presence of high amount of protopanaxadiol ginsenoside as compared to main root part (7).

\subsection{Mechanism of action}

Ginsenosides weave themselves into the plasma membranes. They possess both the hydrophobic and the hydrophilic part, thus they are amphiphilic in nature. This insertion induces changes in cell functions by affecting membrane functions (3). As a result, a dual mode of action was observed. First pathway involves inducing changes in electrolyte transport system when ginsenosides bind to the membrane receptors and activates signal pathways
(3). The second pathway involves ginsenoside binding to intracellular steroid receptors where the transcription occurs through the action of ligand/receptor complex (8).

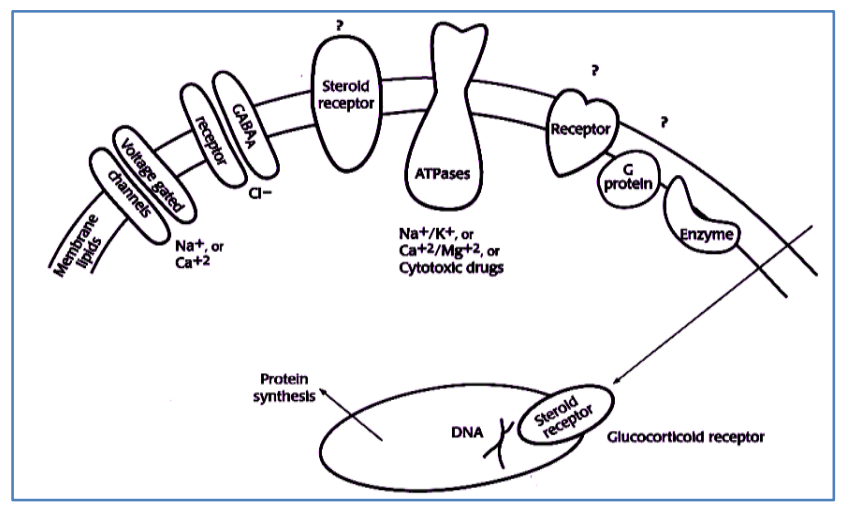

Figure 4: Potential ginsenoside action sites on plasma and nuclear membrane (3)

\subsection{Therapeutic benefits of Ginseng}

\subsection{Anti-carcinogenic effects}

Controlled trials have shown that ginseng showcased number of anti- carcinogenic activities depending on the age and type of ginseng, it is considered to be among functional food to prevent non- organ specific cancers. There was a decreased risk of cancer with increased intake of ginseng $(9,10)$. Chronic inflammation leads to high risk of cancer, therefore, ginseng can be used as an anti inflammatory tool to target inflammation causing agents (11). Ginseng was found to specifically lower the risk of cancer that was caused due to smoking (15).

\subsection{Treatment for Degenerative disorders}

Ginsenosides were found to inhibit the activity of AChE (acetylcholinesterase), BChE (butyrylcholinesterase) and BACE1 which are the enzymes that are responsible for the onset of Alzheimer's disease because of their ability to catalyse the breakdown of neurotransmitters like acetylcholine and some esters (12). It was established that lonesome administration of ginseng didn't have a major effect on AD progression. Although when combined with other $A D$ drugs it may improve the cognitive functions in $A D$ patients (13). Gintonin which is relatively a new component of ginseng was found to improve memory formation in mice when administered systemically (14).

\subsection{Immunity boost therapy}

Ginseng administration leads to stimulatory response of antigen specific antibodies as well as cytokine 
synthesis $(16,19)$. Ginseng extract showcased outstanding antioxidant properties with an observed increment in amount of white blood cells, lymphocytes and spleen cells that produce antibodies (17). Ginseng is known for its property of immune regulation by maintaining immune homeostasis. It enhances the resistance to illness (18).

\subsection{Diabetic therapy}

Not only did the Ginseng root extract exhibited the anti- diabetic effect on genetically diabetic mice but ginseng berry showcased a more potent effect on improving glucose tolerance (20). Although type 1 diabetes remained the main focus of the study, Atelle et.al. observed a markedly reduced glucose disposal in patients with type 2 diabetes on exposure to ginseng berry extract. There was a reduced serum insulin concentration in fasting as well as postprandial state (21).

\subsection{Hypertension therapy}

Ginseng is known to stimulate nitric oxide production (NO) which provide vasoprotective effects in aortic endothelial cells and NO derived from endothelium have shown anti- atherosclerosis properties (22). Administering a sample of people having arterial stiffness with $3 \mathrm{~g} /$ day of ginseng for three months reduced diastolic blood pressure (DBP) of the sample group (23). Addition of ginseng with conventional treatment for patients of Hypertension accompanying diabetes resulted in improvement of arterial stiffness and reduced systolic blood pressure (24). North American ginseng (NAG) lacks a specific ginseng Rg3 which explains its neutral effect on blood pressure, whereas it is contained in Panax ginseng, hence, it shows vasodilating effect (25).

\subsection{Obesity}

Ginsenosides are of two types depending upon their structure- protopanaxadiol (PDG) and protopanaxatriol (PTG). PDG extracted from the leaves of American ginseng showed inhibitory effect against anti-obesity and lipase activities. It showed a notable reduction in serum, tissue and liver triglyceride levels (26).

\begin{tabular}{|c|c|c|c|c|}
\hline Group & $\begin{array}{l}\text { Final body weight (g/mouse) } \\
\text { mean } \pm \text { S.E. }\end{array}$ & $\begin{array}{l}\text { Liver (g/mouse) } \\
\text { mean } \pm S . E \text {. }\end{array}$ & $\begin{array}{l}\text { Spleen (g/mouse) } \\
\text { mean } \pm S . E \text {. }\end{array}$ & $\begin{array}{l}\text { Kidney (g/mouse) } \\
\text { mean } \pm S . E .\end{array}$ \\
\hline Normal & $34.35 \pm 0.312^{* *}$ & $1.58 \pm 0.060^{* *}$ & $0.13 \pm 0.014$ & $0.37 \pm 0.020$ \\
\hline $\mathrm{HF}$ & $37.83 \pm 0.329$ & $2.31 \pm 0.074$ & $0.12 \pm 0.030$ & $0.41 \pm 0.012$ \\
\hline HF plus 0.02\%PDG & $35.84 \pm 0.298^{*}$ & $1.59 \pm 0.018^{* *}$ & $0.13 \pm 0.014$ & $0.39 \pm 0.016$ \\
\hline HF plus 0.05\%PDG & $36.71 \pm 0.816$ & $1.60 \pm 0.087^{* *}$ & $0.13 \pm 0.019$ & $0.39 \pm 0.014$ \\
\hline HF plus $0.05 \% P D G^{a}$ & $36.84 \pm 0.168$ & $1.58 \pm 0.102^{* *}$ & $0.15 \pm 0.020$ & $0.40 \pm 0.021$ \\
\hline
\end{tabular}

Figure 5: Effect of PDG on organ weights of mice fed with normal diet, high fat diet, high fat diet containing 0.02\%PDG high fat diet containing 0.05\%PDG, high fat diet containing 0.05\% PDG for a span of 8 weeks. (26)

Administration of $1.6 \%$ korean ginseng extract with high fat diet resulting in lower levels of mRNA associated with lipogenesis- genes suggesting that the anti- obesity effects of ginseng are due to gene regulation of lipogenesis related genes in adipose tissue and causing a delay in intestinal absorption (27). Ginseng and ginsenosides were found to regulate appetite as well as inhibit lipid synthesis and increase the level of energy consumption by skeletal muscle through activated AMPK pathway (28). Ginseng intake changes the phyla and genera of gut microbiota and weight loss was dependent on the type of microbes in the gut prior to ginseng intake (29).

\subsection{Eye-care}

Several major eye diseases specially, AMD, glaucoma, cataract, and alternative retinal pathologies, are below investigation for potential helpful effects of botanicals. These diseases will result in ocular harm and visual issues primarily through aerophilic stress, inflammation, and ocular pressure. Similarly, the active chemical ingredients in several botanicals contain sturdy antioxidative, medicinal drug, and anti-apoptotic properties. though this review solely addresses a number of the well-studied and customary Ginseng for treatment of ocular diseases, there are various alternative compounds that will facilitate with treatment of those diseases also. 
whereas some Ginseng, like curcumin or quercetin, are the topic of many studies and clinical trials, the advantages of the many compounds haven't been examined as extensively.e have summarized the compounds that are used for either pre-clinical or clinical trials (30). There are many studies that indicated the mechanistic pathways of those compounds as being effective in cellular stress but, intensive characterization continues to be needed to bring these compounds for therapeutic analysis and human clinical trials. Thus, any investigation of natural plant-derived compounds, and particularly their mechanisms of action, is important to harness the total potential of natural compounds to be used as a non-invasive and preventative complementary and/or different for major eye diseases.

\subsection{Cardiovascular care}

This review summarized current info regarding the effectivity of ginseng on major vas risk factors like cardiovascular disease, internal organ illness, hyperlipidaemia, aerophilus stress, and particle regulation. Ginseng may be a ancient seasoning remedy whose antiquity stretches back to precedent days. The active constituent ginsenosides play an important role within the medicative effects of ginseng. Ginsenosides exhibit their immense vary of activities on CVD through the inhibition of ROS production, stimulation of NO production, improvement in blood circulation, improvement of dilation tone, and regulation of the supermolecule profile. However, the precise mechanisms of action of ginsenosides are still unidentified. within the future, every ginsenoside should be studied on its specific mechanism of action on CVD. The common use of ginseng as a seasoning remedy needs strict investigations to assess each its effectivity and its safety (31).

\subsection{Ginseng and estrogen}

Ginseng has been known for thousands of years in the Far East as a stimulant. In recent years ithas attracted interest in western countries. Its primary action, however, remains uncertain. We report anoestrogen-like effect of ginseng on the vaginal epithelium and competition by ginseng saponins with 17-oestradiol and R5020 for binding to human myometrial receptor proteins (32).

\subsection{Neural care}

Many ginsenosides are isolated and characterised. The molecular mechanisms related to ginsenosides involve scavenging free radicals, inhibition of inflammation, and bar of excitotoxicity. Animal and cell culture studies have indicated that ginsenosides have completely different activities in each physiological and pathologic conditions. The structure activity relationship of ginsenosides has not been completely elucidated. However, it's turning into progressively evident that ginsenosides turn out neuroprotective effects by reducing radical production and enhancing brain perform. Studies involving every ginsenoside ought to embody mechanisms of action, specificity, structure and performance relationship, elaborated pharmacology and toxicity studies, and therapeutic studies in animal models and humans. more studies are necessary to look at the consequences of ginseng on metabotropic salt receptors and transporters, and therefore the Keap1/Nrf2 accommodative cellular pathway (33). The medicine disorders that there's most proof from pre- clinical and attiny low range of clinical studies to profit from ginseng are $A D$ and major depression, and clinical trials are necessary to substantiate the effectiveness of ginseng and ginsenosides within the bar and treatment of those, and probably alternative medicine conditions.

\subsection{Depression}

Patients with anxiety and depression can develop a variety of diseases, as discussed above. This is because anxiety and depression can promote inflammatory responses. First, the proinflammatory cytokines such as IL-1, IL-6, interferon- $\gamma$, and TNF- $\alpha$ play a role. Second, oxidative or nitrosative stress can occur owing to the increased levels of reactive oxygen species and reactive nitrogen species. As a result, anxiety and depression can predispose patients to the development of cancers, neurodegenerative conditions, and inflammatory diseases (34).

\section{Future Researches and Side effects}

Future researches should work on ginsenoside partition to see if they produce structural changes in membrane protein (3).

Consumption of doses much higher than the recommended dose $(15 \mathrm{~g} /$ day) resulted in nervousness, insomnia and gastrointestinal disturbance (4). 


\begin{tabular}{|c|c|c|c|}
\hline Adverse effects & Samples & Patients & Types of reports \\
\hline Acute lobular hepatitis & Ginseng energy drinks & 26-yr-old male leukemia patient & Case report \\
\hline Allergy & Asian ginseng syrup. & 20-yr-old male patient & Case report \\
\hline Allergy & Panax ginseng & 29-yr-old female patient & Case report \\
\hline Allergy & Korean ginseng extract & 44-yr-old man & Case report \\
\hline Allergy & Korean ginseng & 34-yr-old woman & Case report \\
\hline Atrial fibrillation with bradycardia & Asian ginseng & 83-yr-old woman with chronic renal insufficiency & Case report \\
\hline Gynecomastia & Red ginseng extract & 12-yr-old boy & Case report \\
\hline Hypertensive crisis & Ginseng Forte-Dietisa & 64-yr-old male who had never suffered hypertension & Case report \\
\hline $\begin{array}{l}\text { Increase in QTc interval and Reduction } \\
\text { of diastolic pressure }\end{array}$ & P. ginseng extract & Total 30 healthy adults & Clinical trial \\
\hline Long QT and torsades de pointes & Large amounts of ginseng drink & 43-yr-old healthy woman & Case report \\
\hline Manic episode & Ginseng root extract & 56-yr-old woman with previous affective disorder & Case report \\
\hline Manic episode & Chinese red ginseng & $\begin{array}{l}\text { 26-yr-old healthy individual with no history of } \\
\text { psychiatric illness }\end{array}$ & Case report \\
\hline Reduction of warfarin action & American ginseng & 20 healthy individuals & Clinical trial \\
\hline Uterine bleeding & $\begin{array}{l}\text { Ginseng cosmetics and ginseng } \\
\text { capsules }\end{array}$ & 39-yr-old woman & Case report \\
\hline Uterine bleeding & Pharmaton Complex & 48-yr-old woman & Case report \\
\hline
\end{tabular}

Figure 6: Risks related with ginseng abuse (35)

\section{References}

1. Taik-Koo Yun, Brief Introduction of Panax ginseng C.A. Meyer, J Korean Med Sci 2001; 16(Suppl): S3-5

2. María L. Escobar-Sánchez, Luis Sánchez-Sánchez and Jesús Sandoval-Ramírez, Steroidal Saponins and Cell Death in Cancer, DOI: 10.5772/61438

3. Anoja S. Attele, Ji An Wu And Chun-su Yuan, Ginseng Pharmacology, Multiple Constituents And Multiple Actions, Biochemical Pharmacology, Vol. 58, Pp. 1685-1693, 1999.

4. C. Norman Gillis, Panax ginseng Pharmacology: A Nitric Oxide Link?, Biochemical Pharmacology, Vol. 54, pp. 1-8, 1997

5. S.K. Kulkarni, A. Dhir / Progress in NeuroPsychopharmacology \& Biological Psychiatry 32 (2008) 1093-1105

6. Soldati F and Sticher O, HPLC separation and quantitative determination of ginsenosides from Panax ginseng, Panax quinquefolium and from ginseng drug preparations. Planta Med 39: 348-357, 1980.

7. Sang Myung Lee, Bong-Seok Bae, Hee-Weon Park, Nam-Geun Ahn, Byung-Gu Cho, Yong-Lae Cho, YiSeong Kwak; Characterization of Korean Red Ginseng (Panax ginseng Meyer): History, preparation method, and chemical composition. J Ginseng Res 39 (2015) 384e391.

8. Chung $E$, Lee $K Y$, Lee $Y J$, Lee $Y H$ and Lee SK, Ginsenoside- $\operatorname{Rg} 1$ down-regulates glucocorticoid receptor and displays syn- ergistic effects with CAMP. Steroids 63: 421-424, 1998.

9. Yun TK and Choi SY, Preventive effect of ginseng intake against various human cancers: A case study on 1987 pairs. Cancer Epidemiol Biomarkers Prev 4: 401-408, 1995.

10. Yun TK, Experimental and epidemiological evidence of the cancer-preventive effects of Panax ginseng C.A. Meyer. Nutr Rev 54: S71-S81, 1996.

11. Lorne J. Hofseth and Michael J. Wargovich; Inflammation, Cancer, and Targets of Ginseng.
12. R.J. Choi, A. Roy, H.J. Jung, M.Y. Ali, B.S. Min, C.H. Park, T. Yokozawa, T.P. Fan, J.S. Choi, H.A. Jung; BACE1 molecular docking and anti-Alzheimer's disease activities of ginsenosides, J Ethnopharmacol, 190 (2016), pp. 219-230, 10.1016/j.jep.2016.06.013

13. Hyeon-Joong Kim, Seok-Won Jung, Seog-Young Kim, Ik-Hyun Cho, Hyoung-Chun Kim, Hyewhon Rhim, Manho Kim, Seung-Yeol Nah; Panax ginseng as an adjuvant treatment for Alzheimer's disease. Volume 42, Issue 4, October 2018, Pages 401-411

14. S. Kim, M.S. Kim, K. Park, H.J. Kim, S.W. Jung, S.Y. Nah, J.S. Han, C. Chung Hippocampus-dependent cognitive enhancement induced by systemic gintonin administration J Ginseng Res, 40 (2016), pp. 55-61, 10.1016/j.jgr.2015.05.001

15. Taik-koo Yun, Soo-Yong Choi; Preventive effect of Ginseng intake against various human cancers: A case control study on 1987 pairs. Cancer epidemiology, biomarker \& prevention. Vol. 4, 401-408, June 1995

16. Soowon Kang and Hyeyoung Min, Ginseng, the 'Immunity Boost': The Effects of Panax ginseng on Immune System. J Ginseng Res Vol. 36, No. 4, 354368 http://dx.doi.org/10.5142/jgr.2012.36.4.354

(2012),

17. A comparative study on immune-stimulatory and antioxidant activities of various types of ginseng extracts in murine and rodent models. Evelyn Saba, Yuan Yee Lee, Minki Kim, Seung-Hyung Kim, SeungBok Hong, Man Hee Rhee. J Ginseng Res 42 (2018) 577e584.

18. Joon-II Lee, Kyoung Sun Park, Ik-Hyun Cho; Panax ginseng: a candidate herbal medicine for autoimmune disease.Journal of Ginseng Research, Volume 43, Issue 3, July 2019, Pages 342-348

19. Maria Waldorff Larsen, Claus Moser, Niels Høiby, Zhijun Song And Arsalan Kharazmi. Ginseng modulates the immune response by induction of interleukin-12 production. APMIS 112: 369-73, 2004

20. Jing-Tian Xie, Sangeeta Mehendale, and Chun-Su Yuan. Ginseng and Diabetes; The American Journal of Chinese Medicine, Vol. 33, No. 3, 397-404 
21. Anoja S. Attele \& Yun-Ping Zhou, Antidiabetic Effects of Panax ginseng Berry Extract and the Identification of an Effective Component; DIABETES, VOL. 51, JUNE 2002

22. Myung-Haeng Hur, Myeong Soo Lee Hye Jeong Yang, Chan Kim , Ik-Lyul Bae, and Edzard Ernst; ginseng for reducing the blood pressure in patients with hypertension: a systematic review and meta-analysis. J. ginseng res. vol. 34, no. 4, 342-347 (2010); doi:10.5142/jgr.2010.34.4.342

23. Moo-Yong Rhee, Yong-Seok Kim, Jun-Ho Bae, DeukYoung Nah, Young-Kwon Kim, Myoung-Mook Lee and Hae-Young Kim; Effect of Korean Red Ginseng on Arterial Stiffness in Subjects with Hypertension. The Journal Of Alternative And Complementary Medicine Volume 17, Number 1, 2011, pp. 45-49. DOI: 10.1089/acm.2010.0065

24. I. Mucalo et al. / Journal of Ethnopharmacology 150 (2013) 148-153

25. P. Mark Stavro, Minna Woo, Tibor F. Heim, Lawrence A. Leiter, Vladimir Vuksan; North American ginseng exerts a neutral effect on blood pressure in individuals with hypertension.

26. Rui Liu, JingZhao Zhang, WenCong Liu, Yoshiyuki Kimura, YiNan Zheng; Anti-Obesity effects of protopanaxdiol types of Ginsenosides isolated from the leaves of American ginseng (Panax quinquefolius L.) in mice fed with a high-fat diet. Fitoterapia 81 (2010) 1079-1087.

27. Young-Sil Lee, Byung-Yoon Cha, Kohji Yamaguchi, Sun-Sil Choi, Takayuki Yonezawa Toshiaki Teruya,
Kazuo Nagai, Je-Tae Woo; Effects of Korean white ginseng extracts on obesity in high- fat diet-induced obese mice. Cytotechnology (2010) 62:367-376. DOI 10.1007/s10616-010-9288-7

28. Zhipeng Li, Geun Eog Ji; Ginseng and obesity. J Ginseng Res 42 (2018) 1-8.

29. Mi-Young Song, Bong-Soo Kim, Hojun Kim; Influence of Panax ginseng on obesity and gut microbiota in obese middle-aged Korean women. J Ginseng Res 38 (2014) 106-115.

30. Jisu Kim, Su-Young Han, Hyeyoung Min; Ginseng for an eye: effects of ginseng on ocular diseases. https://doi.org/10.1016/j.jgr.2018.11.006.

31. Chang HoLee1Jong-HoonKim; A review on the medicinal potentials of ginseng and ginsenosides on cardiovascular diseases. https://doi.org/10.1016/ j.jgr.2014.03.001

32. R Punnonen, A Lukola; British Medical journal. Volume 281. doi: 10.1136/bmj.281.6248.1110

33. Wei-Yi Ong, Tahira Farooqui, Hwee-Ling Koh, Akhlaq A. Farooqui and Eng-Ang Ling; Protective effects of ginseng on neurological disorders. doi: 10.3389/fnagi.2015.00129

34. Seungyeop Lee, Dong-Kwon Rhee; Effects of ginseng on stress-related depression, anxiety, and the hypothalamic-pituitary-adrenal axis. https://doi.org/10.1016/j.jgr.2017.01.010

35. Doo Jin Paik, Chang Ho Lee; Review of cases of patient risk associated with ginseng abuse and misuse J Ginseng Res 39 (2015) 89e93 